\title{
Predicting risk of severe hypoglycaemia in type 2 diabetes
}

\author{
Sankalpa Neupane • Mark L. Evans
}

Received: 17 February 2015 / Accepted: 19 February 2015 /Published online: 21 March 2015

(C) Springer-Verlag Berlin Heidelberg 2015

\begin{abstract}
For many, the extent to which blood glucose control can be lowered is limited by risk of hypoglycaemia. Hypoglycaemia is feared and carries fiscal, social and medical costs, with risk of death being associated with severe hypoglycaemia in patients with type 2 diabetes at high cardiovascular risk. In this issue of Diabetologia, Chow et al (DOI: 10.1007/s00125-015-3512-0) report that patients with type 2 diabetes who suffered severe hypoglycaemia during attempts to lower blood glucose intensively were more likely to be insulin deficient and/or carry markers of autoimmunity more usually associated with type 1 diabetes. This opens the question of whether biomarkers might help clinicians identify those patients at greater or lower risk of treatment-induced hypoglycaemia, allowing therapeutic targets to be modified accordingly.
\end{abstract}

Keywords Autoantibodies $\cdot$ Biomarkers $\cdot$ Hypoglycaemia

\section{Abbreviation \\ ACCORD Action to Control Cardiovascular Risk in Diabetes}

Following the publication of the UK Prospective Diabetes Study (UKPDS) in 1998 [1], glycaemic targets in type 2 diabetes seemed simple. The lower the achieved $\mathrm{HbA}_{1 \mathrm{c}}$, the better in terms of reducing the risk of complications. Hypoglycaemia was generally regarded as an inconvenient obstacle and/or an inevitable by-product of these attempts to lower blood glucose. The $\mathrm{HbA}_{1 \mathrm{c}}$ achieved in the intensive and

\footnotetext{
S. Neupane $\cdot$ M. L. Evans $(\bowtie)$

Wellcome Trust/MRC Institute of Metabolic Science and Department of Medicine, IMS Metabolic Research Laboratories, University of Cambridge, Box 289 Addenbrookes Hospital/Cambridge NIHR Biomedical Research Centre, Hills Road, Cambridge CB2 0QQ, UK e-mail: Mle24@cam.ac.uk
}

standard arms of UKPDS were $53 \mathrm{mmol} / \mathrm{mol}(7.0 \%)$ and $63 \mathrm{mmol} / \mathrm{mol}$ (7.9\%), respectively, so still above the nondiabetic range. Three large studies then set out to examine whether lowering $\mathrm{HbA}_{1 \mathrm{c}}$ further towards the non-diabetic range in type 2 diabetic patients with high cardiovascular risk led to further reductions in complication risk. Two of these, the Action in Diabetes and Vascular Disease: Preterax and Diamicron Modified Release Controlled Evaluation (ADVANCE) and the Veterans Affairs Diabetes Trial (VADT), showed no benefits in terms of macrovascular disease or mortality from tight control $[2,3]$. Alarmingly, the third study, the US-based Action to Control Cardiovascular Risk in Diabetes (ACCORD) study showed increased mortality in the intensively treated group and was halted early [4].

The cause(s) for this apparently paradoxical rise in mortality with tight blood glucose control remain unexplained. However, it was clear that more aggressive blood glucose lowering was associated with increased risk of hypoglycaemia and that there was also a robust association between severe hypoglycaemia — defined as episodes requiring external assistance - and risk of dying in the ACCORD study. The obvious question of whether the relationship between severe hypoglycaemia and mortality is causal or mere association remains undetermined [5]. Although experimental studies in humans and rodents have identified potential mechanistic changes that could explain an association, for example, by inducing cardiac dysrhythmias [6,7], infrequent fatal events are difficult to study at a population level. However, whether it increases the risk of dying or not, hypoglycaemia is still clinically important, being feared by patients as much as chronic complications, increasing the risk of accidents, carrying social and economic costs (e.g. limitations on occupation, driving) and limiting the degree to which $\mathrm{HbA}_{1 \mathrm{c}}$ can be lowered.

We already know from type 1 diabetes that a small subset of patients accounts for a relatively large proportion of hypoglycaemic episodes. Assuming that different groups of 
patients with type 2 diabetes-a more heterogeneous disease - might also show different risks, this could be of relevance to clinical practice. If we could predict in advance which type 2 diabetic patients might be at higher or lower risk of hypoglycaemia, then treatment regimens and the degree of intensification of glycaemia could be individualised accordingly. In this issue of Diabetologia, Chow and colleagues examined a cohort of ACCORD patients for biomarkers associated with severe hypoglycaemia. They compared 326 cases from the ACCORD study who had severe hypoglycaemia despite failure to achieve $\mathrm{HbA}_{1 \mathrm{c}}$ below $42 \mathrm{mmol} / \mathrm{mol}(6.0 \%)$ with demographically matched (for age, race, $\mathrm{BMI}$ ) controls who were able to achieve an $\mathrm{HbA}_{1 \mathrm{c}}$ below $42 \mathrm{mmol} / \mathrm{mol}$ without severe hypoglycaemia [8]. Hypothesising that there is an association between hypoglycaemia and insulinopenia, Chow et al found that baseline insulin deficiency (fasting C-peptide $\leq 0.15 \mathrm{nmol} / \mathrm{l}$ ) was significantly associated with severe hypoglycaemia (OR 23 comparing severe hypoglycaemia cases and non-severe hypoglycaemia controls). There were weaker but significant associations with the presence of detectable autoantibodies more usually associated with type 1 diabetes (glutamic acid decarboxylase [GAD], tyrosine phosphatase-related islet antigen-2 [IA2], zinc transporter 8 [ZnT8]).

There are a number of potential explanations for an association between insulin deficiency and risk of severe hypoglycaemia in type 2 diabetes. First, an obvious possible reason is that patients with insulin deficiency are likely to be treated with insulin, the most potent blood glucose-lowering therapy, with attendant risk of overshooting into hypoglycaemia. In addition, counter-regulatory responses are reduced in type 2 diabetic patients with insulin deficiency. In health, a major counter-regulatory defence against a falling blood glucose is the ability of the healthy beta cell to switch off endogenous insulin secretion. This occurs at an early stage of hypoglycaemia as blood glucose falls below $4 \mathrm{mmol} / \mathrm{l}$. Nearly $90 \%$ of the hypoglycaemia group were on exogenous insulin at baseline, with little or no ability to modulate circulating insulin as glucose levels fell. In addition to switching off insulin, the healthy islet will increase glucagon release in response to a falling blood glucose level. Glucagon counterregulatory responses to hypoglycaemia are also thought to become deficient in long-standing type 2 diabetes with beta cell failure/ exhaustion.

Approximately $10 \%$ of patients diagnosed clinically with type 2 diabetes have detectable islet antibodies [9, 10], a condition attracting various labels, including latent autoimmune diabetes in adults (LADA). The assumption here is that the association between severe hypoglycaemia and markers of autoimmunity is mediated by reduced insulin secretion. In addition to autoimmunity, insulin deficiency may develop with long-standing type 2 diabetes and, consistent with this, the severe hypoglycaemia group studied here had longer duration of known diabetes than their demographically matched controls. These observations are in keeping with data reporting that rates of severe hypoglycaemia in long-standing type 2 diabetes are similar to those seen in type 1 diabetes [11].

Leaving aside the issues raised from the main ACCORD findings about what glycaemic targets to aim for, what are the possible implications of this work for clinicians and patients wanting to intensify blood glucose lowering but concerned about the risk of hypoglycaemia? First, this confirms clinical suspicion that there may be 'biological' determinants of hypoglycaemia risk. In other words, even when allowing for known hypoglycaemia risk factors better defined in type 1 diabetes (e.g. alcohol, dose errors, missed meals, activity, concurrent illness, therapy concordance), there may still be intrinsic reasons why some groups of patients are just at higher risk of hypoglycaemia with intensified glucose lowering.

Intriguingly, there have been reports of severe hypoglycaemia risk being associated with a polymorphism $(\mathrm{D} / \mathrm{D}$ deletion) in the gene encoding angiotensin-converting enzyme (ACE) in both type 2 and type 1 diabetes $[12,13]$. The postulated mechanism here is different from those described above, with molecular differences in the renin-angiotensin system determining how sensitive cognitive functioning is to a fall in glucose. Those carrying the DD ACE genotype, for example, are thought to be more likely to develop cognitive dysfunction as blood glucose falls and brain fuel supply becomes impaired. This means that judgement and the ability to make rational decisions about self-treatment of hypoglycaemia may become affected earlier and/or more profoundly during hypoglycaemia, increasing the chances that external assistance is needed for rescue. This illustrates the point that different causes may exist for severe hypoglycaemia risk: therapeutic, educational, behavioural, biological and just plain bad luck/circumstances. In large longitudinal studies, better phenotyping of the nature and potential drivers of hypoglycaemia may be needed for identification of robust biomarkers, perhaps allowing therapies to be targeted accordingly.

Should we now be routinely measuring autoantibodies and insulin reserve in type 2 diabetes to predict severe hypoglycaemia risk? The answer is probably not, based on these data. This study was a post hoc analysis based on a cohort of ACCORD patients identified because of their glycaemic responses and did not examine the predictive value for clinicians to use these biomarkers to predict severe hypoglycaemia risk. Looking at the distribution of the biomarkers, $17 \%$ of patients experiencing severe hypoglycaemia had insulin deficiency, with a similar number having antiGAD antibodies. This means that $83 \%$ of patients experiencing severe hypoglycaemia would not have been identified by either of these measures alone. Further work is needed to establish the merits of screening for insulin deficiency in type 2 diabetes, but this is an important clinical area. 
Individualising treatment regimens is of course already the stock trade of clinical teams. For example, the choice of therapy and glycaemic targets will probably be different in an 85 year-old with type 2 diabetes compared with a 40 -year-old patient. All of this emphasises that type 2 diabetes is a mixed bag and that clinical management should be individualisedan important point that can easily be lost with generic type 2 diabetes clinical guidelines!

Funding SN is supported by the NIHR Cambridge Biomedical Centre.

Duality of interest The authors declare that there is no duality of interest associated with this manuscript.

Contribution statement Both authors were responsible for the design of the manuscript, drafting the article and revising it critically for important intellectual content. Both authors approved the version to be published.

\section{References}

1. UK Prospective Diabetes Study (UKPDS) Group (1998) Intensive blood-glucose control with sulphonylureas or insulin compared with conventional treatment and risk of complications in patients with type 2 diabetes (UKPDS 33). Lancet 352:837-853

2. The Action to Control Cardiovascular Risk in Diabetes Study Group (2008) Effects of intensive glucose lowering in type 2 diabetes. N Engl J Med 358:2545-2559

3. Duckworth W, Abraira C, Moritz T et al (2009) Glucose control and vascular complications in veterans with type 2 diabetes. $\mathrm{N}$ Engl $\mathrm{J}$ Med 360:129-139
4. The ADVANCE Collaborative Group (2008) Intensive blood glucose control and vascular outcomes in patients with type 2 diabetes. $\mathrm{N}$ Engl J Med 358:2560-2572

5. Bonds DE, Miller ME, Bergenstal RM et al (2010) The association between symptomatic, severe hypoglycaemia and mortality in type 2 diabetes: retrospective epidemiological analysis of the ACCORD study. BMJ 340:b4909

6. Chow E, Bernjak A, Williams S et al (2014) Risk of cardiac arrhythmias during hypoglycemia in patients with type 2 diabetes and cardiovascular risk. Diabetes 63:1738-1747

7. Reno CM, Daphna-Iken D, Chen YS, Van der Weele J, Jethi K, Fisher SJ (2013) Severe hypoglycemia-induced lethal cardiac arrhythmias are mediated by sympathoadrenal activation. Diabetes 62:3570-3581

8. Chow LS, Chen H, Miller ME, Marcovina SM, Seaquist ER (2015) Biomarkers related to severe hypoglycaemia and lack of good glycaemic control in ACCORD. Diabetologia. doi:10.1007/s00125015-3512-0

9. Irvine WJ, Gray RS, McCallum CJ, Duncan LJP (1977) Clinical and pathogenic significance of pancreatic-islet-cell antibodies in diabetics treated with oral hypoglycaemic agents. Lancet 1:1025-1027

10. Hawa MI, Buchan AP, Ola T et al (2014) LADA and CARDS: a prospective study of clinical outcome in established adult-onset autoimmune diabetes. Diabetes Care 37:1643-1649

11. Hypoglycaemia Study Group UK (2007) Risk of hypoglycaemia in types 1 and 2 diabetes: effects of treatment modalities and their duration. Diabetologia 50:1140-1147

12. Davis WA, Brown SGA, Jacobs IG et al (2011) Angiotensinconverting enzyme insertion/deletion polymorphism and severe hypoglycemia complicating type 2 diabetes: the Fremantle Diabetes Study. J Clin Endocrinol Metab 96:E696-E700

13. Pedersen-Bjergaard U, Agerholm-Larsen B, Pramming S, Hougaard P, Thorsteinsson B (2003) Prediction of severe hypoglycaemia by angiotensin-converting enzyme activity and genotype in type 1 diabetes. Diabetologia 46:89-96 